Rabha W. Ibrahim*, Dumitru Baleanu, and Jay M. Jahangiri

\title{
Conformable differential operators for meromorphically multivalent functions
}

https://doi.org/10.1515/conop-2020-0113

Received March 2, 2021; accepted March 8, 2021

Abstract: We define a conformable diff-integral operator for a class of meromorphically multivalent functions. We show that this conformable operator adheres to the semigroup property. We then use the subordination properties to prove inclusion conditions, sufficienrt inclusion conditions and convolution properties for this class of conformable operators.

Keywords: Subordination and superordination, meromorphically multivalent functions, univalent function, analytic function, open unit disk, conformable fractional operator, fractional calculus

PACS: 30C55, 30C45

\section{Introduction}

The differential and integral (diff-integral) calculus of integer-order developed by Leibniz and Newton was a great discovery in mathematics, having numerous applications in several areas of physics, biology, engineering and others. The idea of the derivative of non-integer oder (commonly known as fractional calculus) was motivated by the question, "What is $\frac{d^{n}}{d t^{n}} f(t)$ for $n=\frac{1}{2}$ and $t$ a real variable?", first initiated by Liebniz in his 1695 letter to L'Hospital which was not surfaced or published until 1849 [1]. Several types of fractional derivatives have been introduced to date, among which are the Riemann-Liouville, Caputo, Hadamard, Caputo-Hadamard, Riesz and other types [2].

The fractional differential operators usually characterize physical capacities, the derivatives signify their proportions of modification, and the operator expresses a connection between them. Because such relations are exceptionally common, differential operators play a pivotal role in many areas of science. Recently, a new local and limit-based extension of fractional derivatives, called conformable fractional derivative, has been formulated by Khalil et al. [3], Katugampola [4], and Abdeljawad [5].

In 2015, Anderson and Ulness [6] introduced a conformable differential operator (CDO) which can be used for control system analysis and design (also see [7]). In 2019, Ibrahim and Jahangiri [8] introduced a conformable differential operator for a class of analytic functions $g$ which are to be univalent in the open unit disk $U=\{z \in \mathbb{C}:|z|<1\}$. Very recently, Ibrahim and Baleanu $[9,10]$ used the operator given in [8] to formulate a hybrid conformable diff-integral operator and a quantum hybrid operator respectively. In addition, fractional differential and integral operators are used to generalize different concepts in information science such as entropy [11, 12].

`Corresponding Author: Rabha W. Ibrahim: IEEE: 94086547, Kuala Lumpur, 59200, Malaysia,

E-mail: rabhaibrahim@yahoo.com

Dumitru Baleanu: Department of Mathematics, Cankaya University, 06530 Balgat, Ankara, Turkey, Institute of Space Sciences, R76900 Magurele-Bucharest, Romania, Department of Medical Research, China Medical University, Taichung 40402, Taiwan, E-mail: dumitru@cankaya.edu.tr

Jay M. Jahangiri: Mathematical Sciences, Kent State University, Burton, Ohio 44021-9500, U.S.A., E-mail: jjahangi@kent.edu 
In this paper we extend the conformable derivative operator defined in [8] to the class of meromorphic functions $\Sigma_{k}(\rho)$ consisting of functions $f$ with the power series expansion

$$
f(z)=z^{-\rho}+\sum_{n=k}^{\infty} a_{n} z^{n-\rho}, \quad z \in U,
$$

where $k \in \mathbb{N}=\{1,2,3, \ldots\}$ and $n-\rho \in \mathbb{N}$. Recall that the functions $f$ of the form (1.1) are called meromorphic with a pole at $z=0$ so that $f(z)-z^{-\rho}$ is analytic in $U$ (see Komatu [13] or Hayman [14]). We then concentrate on a subclass of $\Sigma_{k}(\rho)$ defined by a subordination and prove inclusion properties and sufficient inclusion conditions for this class and examin its closure property under convolution or Hadamard product.

\section{Preliminaries}

In this section we state a few definitions and a lemma that we shall need in the next section. First, we define a conformable differential operator for the class of meromorphic functions $\Sigma_{k}(\rho)$ defined by (1.1).

Definition 2.1. For functions $f \in \Sigma_{k}(\rho)$, define the conformable differential operator as follows:

$$
\begin{aligned}
& \Delta^{0} f(z)=f(z), \\
& \begin{aligned}
\Delta^{v} f(z)= & \frac{\lambda_{1}(v, z)}{\lambda_{1}(v, z)+\lambda_{0}(v, z)} f(z)+\frac{\lambda_{0}(v, z)}{\lambda_{1}(v, z)+\lambda_{0}(v, z)}\left(\frac{-z}{\rho}\right) f^{\prime}(z) \\
& =z^{-\rho}+\sum_{n=k}^{\infty} a_{n}\left(\frac{\lambda_{1}(v, z)+((\rho-n) / \rho) \lambda_{0}(v, z)}{\lambda_{1}(v, z)+\lambda_{0}(v, z)}\right) z^{n-\rho}, \\
\Delta^{2 v} f(z)= & \Delta\left(\Delta^{v} f(z)\right) \\
& =z^{-\rho}+\sum_{n=k}^{\infty} a_{n}\left(\frac{\lambda_{1}(v, z)+((\rho-n) / \rho) \lambda_{0}(v, z)}{\lambda_{1}(v, z)+\lambda_{0}(v, z)}\right)^{2} z^{n-\rho}, \\
\vdots & \\
\Delta^{m v} f(z)= & \Delta^{v}\left[\Delta^{(m-1) v} f(z)\right] \\
& =z^{-\rho}+\sum_{n=k}^{\infty} a_{n}\left(\frac{\lambda_{1}(v, z)+((\rho-n) / \rho) \lambda_{0}(v, z)}{\lambda_{1}(v, z)+\lambda_{0}(v, z)}\right)^{m} z^{n-\rho} \\
& =z^{-\rho}+\sum_{n=k}^{\infty} a_{n}\left(\Lambda_{n}\right)^{m} z^{n-\rho},
\end{aligned}
\end{aligned}
$$

where $v \in[0,1], \rho \in \mathbb{N}, m \in \mathbb{N} \cup\{0\}, z \in U$,

$$
\lim _{v \rightarrow 0} \lambda_{1}(v, z)=1, \quad \lim _{v \rightarrow 1} \lambda_{1}(v, z)=0, \quad \lambda_{1}(v, z) \neq 0, \forall z \in U^{\star}, v \in(0,1),
$$

and

$$
\lim _{v \rightarrow 0} \lambda_{0}(v, z)=0, \quad \lim _{v \rightarrow 1} \lambda_{0}(v, z)=1, \quad \lambda_{0}(v, z) \neq 0, \forall z \in U^{\star} v \in(0,1)
$$


Clearly, $\Delta^{m v} f(z) \in \Sigma_{k}(\rho)$ as well as

$$
\begin{aligned}
& \Delta^{m v}[\alpha f(z)+\beta g(z)] \\
& =\left(\frac{\lambda_{1}(v, z)}{\lambda_{1}(v, z)+\lambda_{0}(v, z)}\right)[\alpha f(z)+\beta g(z)] \\
& +\left(\frac{\lambda_{0}(v, z)}{\lambda_{1}(v, z)+\lambda_{0}(v, z)}\right)\left(\frac{-z}{\rho}\right)[\alpha f(z)+\beta g(z)]^{\prime} \\
& =\alpha\left(\left(\frac{\lambda_{1}(v, z)}{\lambda_{1}(v, z)+\lambda_{0}(v, z)}\right) f(z)+\left(\frac{\lambda_{0}(v, z)}{\lambda_{1}(v, z)+\lambda_{0}(v, z)}\right)\left(\frac{-z}{\rho}\right) f^{\prime}(z)\right) \\
& +\beta\left(\left(\frac{\lambda_{1}(v, z)}{\lambda_{1}(v, z)+\lambda_{0}(v, z)}\right) g(z)+\left(\frac{\lambda_{0}(v, z)}{\lambda_{1}(v, z)+\lambda_{0}(v, z)}\right)\left(\frac{-z}{\rho}\right) g^{\prime}(z)\right) \\
& =\alpha \Delta^{m v} f(z)+\beta \Delta^{m v} g(z) ; \alpha, \beta \in \mathbb{R} .
\end{aligned}
$$

So, we proved the following proposition.

Proposition 2.2. (semigroup property) The class of conformal differential operators defined by $\Delta^{m v}$ has the semigroup property since for $f$ and $g$ in $\Sigma_{k}(\rho)$ we have

$$
\Delta^{m v}[\alpha f(z)+\beta g(z)]=\alpha \Delta^{m v} f(z)+\beta \Delta^{m v} g(z) .
$$

We shall need the following subordination definition for our next class of meromorphic functions. For functions $f$ and $g$ in $\Sigma_{k}(\rho)$, we say that $f$ is subordinate to $g$, denoted by $f \prec g$ if there exists a Schwarz function $\omega$ with $\omega(0)=0$ and $|\omega(z)| \leq|z|<1$ so that $f(z)=g(\omega(z))$ in $U$ (see [15] or [18]).

Definition 2.3. For $-1 \leq B<A \leq 1$ and $\sigma<0$, a function $f \in \Sigma_{k}(\rho)$ is said to be in the class $\Sigma_{k}^{v}(A, B, \sigma, \rho)$ if it satisfies the subordination condition

$$
\Psi(z)=(1-\sigma) z^{\rho}\left[\Delta^{m v} f(z)\right]-\left(\frac{\sigma}{\rho}\right) z^{1+\rho}\left[\Delta^{m v} f(z)\right]^{\prime} \prec J_{A, B}(z)=\frac{1+A z}{1+B z} .
$$

The class of functions $J_{A, B}(\omega(z)):=\frac{1+A \omega(z)}{1+B \omega(z)}$ and in particular, the functions of the form $J_{A, B}(z)=\frac{1+A z}{1+B z}$ are of special significance since $J_{A, B}(\omega(z))$ is the class of Caratheodory functions of order $\frac{1-A}{1-B}$, that is, $\Re\left\{J_{A, B}(\omega(z))\right\}>\frac{1-A}{1-B}$ (see Janowski [16] or Jahangiri et. al. [17]).

To prove our results in the next section, we shall also need the following lemma which is due to Miller and Mocanu [18].

Lemma 2.4. Suppose that $\phi(z)$ is analytic in $U$ and $\psi(z)$ is convex univalent in $U$ such that $\phi(0)=\psi(0)$. If $\phi(z)+(1 / \wp) \phi^{\prime}(z) \prec \psi(z)$ for a non-zero complex constant number $\wp$ with $\Re(\wp) \geq 0$, then $\phi(z) \prec \psi(z)$.

\section{Main Results}

First we prove an inclusion theorem for the class $\Sigma_{k}^{v}(A, B, \sigma, \rho)$.

Theorem 3.1. Let $f \in \Sigma_{k}(\rho)$. If $\sigma_{2}<\sigma_{1}<0$ then

$$
\Sigma_{k}^{v}\left(A, B, \sigma_{2}, \rho\right) \subset \Sigma_{k}^{v}\left(A, B, \sigma_{1}, \rho\right) .
$$

Proof. Let $f \in \Sigma_{k}^{\nu}\left(A, B, \sigma_{2}, \rho\right)$. Define a function $\phi(z)=z^{\rho}\left[\Delta^{m v} f(z)\right]$, which is analytic in $U$ with $\phi(0)=1$. A computation implies

$$
\left(1-\sigma_{2}\right) z^{\rho}\left[\Delta^{m v} f(z)\right]-\left(\frac{\sigma_{2}}{\rho}\right) z^{1+\rho}\left[\Delta^{m v} f(z)\right]^{\prime}=\phi(z)-\frac{\sigma_{2}}{\rho}\left(z \phi^{\prime}(z)\right) .
$$


Thus, we obtain the inequality

$$
\phi(z)-\frac{\sigma_{2}}{\rho}\left(z \phi^{\prime}(z)\right) \prec \frac{A z+1}{B z+1} .
$$

Applying Lemma 2.4 with $\wp:=-\frac{\sigma_{2}}{\rho}>0$ yields

$$
\phi(z) \prec \frac{A z+1}{B z+1}, \quad z \in U .
$$

Since, $0<\sigma_{1} / \sigma_{2}<1$ and since $J_{A, B}(z)$ is convex univalent in $U$, we have the following inequality

$$
\begin{aligned}
& \left(1-\sigma_{1}\right) z^{\rho}\left[\Delta^{m v} f(z)\right]-\left(\frac{\sigma_{1}}{\rho}\right) z^{1+\rho}\left[\Delta^{m v} f(z)\right]^{\prime} \\
& =\left(1-\sigma_{1}\right) \phi(z)-\left(\frac{\sigma_{1}}{\rho}\right)\left(z \phi^{\prime}(z)-\rho \phi(z)\right)+\left(\frac{\sigma_{1}}{\sigma_{2}} \phi(z)-\frac{\sigma_{1}}{\sigma_{2}} \phi(z)\right) \\
& =\frac{\sigma_{1}}{\sigma_{2}}\left(\left(1-\sigma_{2}\right) \phi(z)-\left(\frac{\sigma_{2}}{\rho}\right)\left(z \phi^{\prime}(z)-\rho \phi(z)\right)\right)+\left(1-\frac{\sigma_{1}}{\sigma_{2}}\right) \phi(z) \\
& =\frac{\sigma_{1}}{\sigma_{2}}\left(\left(1-\sigma_{2}\right) z^{\rho}\left[\Delta^{m v} f(z)\right]-\left(\frac{\sigma_{2}}{\rho}\right) z^{1+\rho}\left[\Delta^{m v} f(z)\right]^{\prime}\right)+\left(1-\frac{\sigma_{1}}{\sigma_{2}}\right) \phi(z) \\
& \prec J_{A, B}(z) .
\end{aligned}
$$

Therefore, by Definition 2.3, we have $f \in \Sigma_{k}^{v}\left(A, B, \sigma_{1}, \rho\right)$.

Next we prove a sufficient inclusion condition for the class $\Sigma_{k}^{v}(A, B, \sigma, \rho)$.

Theorem 3.2. Let $f \in \Sigma_{k}(\rho)$ and

$$
\Psi(z)=(1-\sigma) z^{\rho}\left[\Delta^{m v} f(z)\right]-\left(\frac{\sigma}{\rho}\right) z^{1+\rho}\left[\Delta^{m v} f(z)\right]^{\prime} .
$$

Then $\Psi(z) \prec J_{A, B}(z)=\frac{1+A z}{1+B z}$ if one of the following inequalities hold

(a) $1+\ell\left(z \Psi^{\prime}(z)\right) \prec \sqrt{z+1}, \ell \geq \max \left\{\ell_{0}, \ell_{1}\right\}$, where

$$
\ell_{0}=\frac{2(0.22599 B+0.22599)}{(A-B)}, \quad B+1 \neq 0, A-B \neq 0 ;
$$

and

$$
\ell_{1}=\frac{2((B-1)(\log (2)-1))}{(A-B)}, B-1 \neq 0, A-B \neq 0
$$

(b) $1+\ell\left(z \frac{\Psi^{\prime}(z)}{\Psi(z)}\right) \prec \sqrt{z+1}, \ell \geq \max \left\{\left|\ell_{2}\right|,\left|\ell_{3}\right|\right\}$, where

$$
\ell_{2}=\frac{-2(i(\log (2)-1))}{2 \pi n-i \log \left(\frac{A-1}{B-1}\right)} ; \log \left(\frac{A-1}{B-1}\right)+2 i \pi n \neq 0, A \neq 1, B \neq 1
$$

and

$$
\begin{gathered}
\ell_{3}=\frac{2 i(-1+\sqrt{2}+\log (2)-\log (1+\sqrt{2}))}{2 \pi n-i \log \left(\frac{B+1}{A+1}\right)}, \\
\left(B+1 \neq 0, A+1 \neq 0, \log \left(\frac{B+1}{A+1}\right)+2 \pi n i \neq 0\right)
\end{gathered}
$$

(c) $1+\ell\left(z \frac{\Psi^{\prime}(z)}{\Psi^{2}(z)}\right) \prec \sqrt{z+1}, \ell \geq \max \left\{\ell_{4}, \ell_{5}\right\}$, where

$$
\begin{gathered}
\ell_{4}=\frac{0.451974(A+1)}{(A-B)}, B+1 \neq 0, A \neq B ; \\
\ell_{5}=\frac{2((A-1)(\log (2)-1))}{(A-B)}, \quad B-1 \neq 0 A \neq B .
\end{gathered}
$$


Proof. Case I: $k=0 \Rightarrow 1+\ell\left(z \Psi^{\prime}(z)\right) \prec \sqrt{z+1}$.

Define a function $F_{\ell}: U \rightarrow \mathbb{C}$ admitting the structure

$$
F_{\ell}(z)=1+\frac{2}{\ell}(\sqrt{z+1}-\log (1+\sqrt{z+1})-1+\log (2)) .
$$

Clearly, $F_{\ell}(z)$ is analytic in $U$ satisfying $F_{\ell}(0)=1$ and it is a solution of the differential equation

$$
1+\ell\left(z F_{\ell}^{\prime}(z)\right)=\sqrt{z+1} .
$$

Therefore, this yields $\mathfrak{W}(z):=\ell\left(z F_{\ell}^{\prime}(z)\right)=\sqrt{z+1}-1$ is starlike in $U$. So for

$$
\mathfrak{F}(z):=\mathfrak{W}(z)+1,
$$

we have

$$
\Re\left(\frac{z \mathfrak{W}^{\prime}(z)}{\mathfrak{W}(z)}\right)=\Re\left(\frac{z \mathfrak{F}^{\prime}(z)}{\mathfrak{W}(z)}\right)>0 .
$$

Thus, by Lemma 2.4 it follows that

$$
1+\mu\left(z \Psi^{\prime}(z)\right) \prec 1+\ell z F_{\ell}^{\prime}(z) \Rightarrow \Psi(z) \prec F_{\ell}(z) .
$$

To conclude this argument, we must show that $F_{\ell}(z) \prec J_{A, B}(z)$. Evidently, the function $F_{\ell}(z)$ is increasing in the interval $(-1,1)$ that is fulfilling the inequality

$$
F_{\ell}(-1) \leq F_{\ell}(1) .
$$

Since

$$
\frac{1-A}{1-B} \leq F_{\ell}(-1) \leq F_{\ell}(1) \leq \frac{1+A}{1+B}
$$

whenever $\ell \geq \max \left\{\ell_{0}, \ell_{1}\right\}$ where

$$
\ell_{0}=\frac{2(B-1)(\log (2)-1)}{(A-B)}, \quad B+1 \neq 0, B-1 \neq 0, A-B \neq 0
$$

and

$$
\ell_{1}=\frac{2(0.225987 B+0.225987)}{(A-B)}, \quad B+1 \neq 0, B-1 \neq 0, A-B \neq 0 .
$$

Consequently, we obtain

$$
\Psi(z) \prec F_{\ell}(z) \prec J_{A, B}(z) \Rightarrow \Psi(z) \prec J_{A, B}(z) .
$$

Case II: $k=1 \Rightarrow 1+\ell\left(\frac{z \Psi^{\prime}(z)}{\Psi(z)}\right) \prec \sqrt{z+1}$.

Define a function $S_{\ell}: U \rightarrow \mathbb{C}$ formulating the structure

$$
S_{\ell}(z)=\exp \left(\frac{2}{\ell}(\sqrt{z+1}-\log (1+\sqrt{z+1})-1+\log (2))\right) .
$$

Obviously, $S_{\ell}(z)$ is analytic in $U$ satisfying $S_{\ell}(0)=1$ and it is an outcome of the differential equation

$$
1+\ell\left(\frac{z S_{\ell}^{\prime}(z)}{S_{\ell}(z)}\right)=\sqrt{z+1}, \quad z \in U .
$$

By assuming $\mathfrak{W}(z)=\sqrt{z+1}-1$, which is starlike in $U$ and $\mathfrak{F}(z)=\mathfrak{W}(z)+1$, we get

$$
\Re\left(\frac{z \mathfrak{W}^{\prime}(z)}{\mathfrak{W}(z)}\right)=\Re\left(\frac{z \mathfrak{F}^{\prime}(z)}{\mathfrak{W}(z)}\right)>0 .
$$

Then again, in virtue of the Lemma 2.4, we obtain

$$
1+\mu\left(\frac{z \Psi^{\prime}(z)}{\Psi(z)}\right) \prec 1+\ell\left(\frac{z S_{\ell}^{\prime}(z)}{S_{\ell}(z)}\right) \Rightarrow \Psi(z) \prec S_{\ell}(z) .
$$




\section{Consequently}

$$
\frac{1-A}{1-B} \leq S_{\ell}(-1) \leq S_{\ell}(1) \leq \frac{1+A}{1+B}
$$

whenever $\ell \geq \max \left\{\left|\ell_{2}\right|,\left|\ell_{3}\right|\right\}$ where

$$
\ell_{2}=\frac{-2(i(\log (2)-1))}{2 \pi n-i \log \left(\frac{A-1}{B-1}\right)} ; \log \left(\frac{A-1}{B-1}\right)+2 i \pi n \neq 0, A \neq 1, B \neq 1
$$

and

$$
\begin{aligned}
& \ell_{3}=\frac{(2 i(-1+\sqrt{(2)}+\log (2)-\log (1+\sqrt{(2)})))}{2 \pi n-i \log \left(\frac{B+1}{A+1}\right)}, \\
& \left(B+1 \neq 0, A+1 \neq 0, \log \left(\frac{B+1}{A+1}\right)+2 \pi n i \neq 0\right)
\end{aligned}
$$

This indicates the following subordination inequalities

$$
\Psi(z) \prec S_{\ell}(z) \prec J_{A, B}(z) \Rightarrow \Psi(z) \prec J_{A, B}(z) .
$$

Case III: $k=2 \Rightarrow 1+\ell\left(\frac{z \Psi^{\prime}(z)}{\Psi^{2}(z)}\right) \prec \sqrt{z+1}$.

Define a function $Q_{\ell}: U \rightarrow \mathbb{C}$ by the formula

$$
Q_{\ell}(z)=\left(1-\frac{2}{\ell}(\sqrt{z+1}-\log (1+\sqrt{z+1})-1+\log (2))\right)^{-1} .
$$

Clearly, $Q_{\ell}(z)$ is analytic in $U$ achieving $Q_{\ell}(0)=1$ and it is the outcome of the differential equation

$$
1+\mu\left(\frac{z Q_{\ell}^{\prime}(z)}{Q_{\ell}(z)}\right)=\sqrt{z+1}
$$

By applying the functions $\mathfrak{W}(z)=\sqrt{z+1}-1$, which is starlike in $U$ and $\mathfrak{F}(z)=\mathfrak{W}(z)+1$, we receive

$$
\Re\left(\frac{z \mathfrak{W}^{\prime}(z)}{\mathfrak{W}(z)}\right)=\Re\left(\frac{z \mathfrak{F}^{\prime}(z)}{\mathfrak{W}(z)}\right)>0 .
$$

Hence, the Lemma 2.4 yields

$$
1+\ell\left(\frac{z \Psi^{\prime}(z)}{\Psi^{2}(z)}\right) \prec 1+\ell\left(\frac{z Q_{\ell}^{\prime}(z)}{Q_{\ell}^{2}(z)}\right) \Rightarrow \Psi(z) \prec Q_{\ell}(z) .
$$

Accordingly, we have

$$
\frac{1-A}{1-B} \leq Q_{\ell}(-1) \leq Q_{\ell}(1) \leq \frac{1+A}{1+B}
$$

if $\ell_{2}$ assumes the upper and lower bounds

$$
\begin{gathered}
\ell_{4}=\frac{0.451974(A+1)}{(A-B)}, B+1 \neq 0, A \neq B ; \\
\ell_{5}=\frac{2((A-1)(\log (2)-1))}{(A-B)}, \quad B-1 \neq 0 A \neq B .
\end{gathered}
$$

This yields the subordination

$$
\Psi(z) \prec Q_{\ell}(z) \prec J_{A, B}(z) \Rightarrow \Psi(z) \prec J_{A, B}(z) .
$$

As a conclusion, we obtain

$$
(1-\sigma) z^{\rho}\left[\Delta^{m v} f(z)\right]-\left(\frac{\sigma}{\rho}\right) z^{1+\rho}\left[\Delta^{m v} f(z)\right]^{\prime} \prec J_{A, B}(z),
$$

for all $\sigma<0$ and $\rho \in \mathbb{N}$. Consequently, $f \in \Sigma_{k}^{v}(A, B, \sigma, \rho)$.

Finally, we prove a convolution condition for the class $\Sigma_{k}^{v}(A, B, \sigma, \rho)$. 
Definition 3.3. The Hadamard product or convolution of two power series

$$
f(z)=z^{-\rho}+\sum_{n=k}^{\infty} a_{n} z^{n-\rho}
$$

and

$$
g(z)=z^{-\rho}+\sum_{n=k}^{\infty} b_{n} z^{n-\rho}
$$

in $\Sigma_{k}(\rho)$ is denoted by

$$
(f \star g)(z)=f(z) \star g(z)=z^{-\rho}+\sum_{n=k}^{\infty} a_{n} b_{n} z^{n-\rho} .
$$

Theorem 3.4. Let $f \in \Sigma_{k}^{v}(A, B, \sigma, \rho)$ and $\varphi \in \Sigma_{k}(\rho)$. Then $f^{\star} \varphi \in \Sigma_{k}^{v}(A, B, \sigma, \rho)$ if

$$
\Re\left(z^{\rho} \Delta^{m v} \varphi(z)\right)>\frac{1}{2} .
$$

Proof. By the properties of the Hadamard product, we have

$$
\begin{aligned}
& (1-\sigma) z^{\rho}\left[\Delta^{m v}\left(f^{\star} \varphi\right)(z)\right]-\left(\frac{\sigma}{\rho}\right) z^{1+\rho}\left[\Delta^{m v}\left(f^{\star} \varphi\right)(z)\right]^{\prime} \\
& =(1-\sigma)\left(z^{\rho}\left[\Delta^{m v} f(z)\right] \star z^{\rho}\left[\Delta^{m v} \varphi(z)\right]\right) \\
& -\left(\frac{\sigma}{\rho}\right)\left(z^{1+\rho}\left[\Delta^{m v} f(z)\right]^{\prime} \star\left(z^{\rho}\left[\Delta^{m v} \varphi(z)\right]\right)\right) \\
& =\left((1-\sigma) z^{\rho}\left[\Delta^{m v} f(z)\right]-\left(\frac{\sigma}{\rho}\right) z^{1+\rho}\left[\Delta^{m v} f(z)\right]^{\prime}\right) \star\left(z^{\rho} \Delta^{m v} \varphi(z)\right) \\
& =\Psi(z)^{\star}\left(z^{\rho} \Delta^{m v} \varphi(z)\right),
\end{aligned}
$$

where $\Psi(z) \prec J_{A, B}(z)$. Given the condition (3.4) yields $\left(z^{\rho} \Delta^{m v} \varphi(z)\right)$ has the Herglotz representation (e.g. see [19])

$$
\left(z^{\rho} \Delta^{m v} \varphi(z)\right)=\int_{|\chi|=1} \frac{d \mu(\chi)}{1-\chi z},
$$

where $d \mu$ indicates the probability measure on the unit circle $|\chi|=1$ and

$$
\int_{|\chi|=1} d \mu(\chi)=1 .
$$

Since $J_{A, B}(z)$ is convex in $U$, we conclude that

$$
\begin{aligned}
& (1-\sigma) z^{\rho}\left[\Delta^{m v}(f \star \varphi)(z)\right]-\left(\frac{\sigma}{\rho}\right) z^{1+\rho}\left[\Delta^{m v}\left(f^{\star} \varphi\right)(z)\right]^{\prime} \\
& =\Psi(z)^{\star}\left(z^{\rho} \Delta^{m v} \varphi(z)\right) \\
& =\int_{|\chi|=1} \Psi(\chi z) d \mu(\chi) \\
& \prec J_{A, B}(z) .
\end{aligned}
$$

Hence, $f^{\star} \varphi \in \Sigma_{k}^{v}(A, B, \sigma, \rho)$.

\section{Conclusion}

From what presented above, it is apparent that we formulated a new conformable differential operator for a class of meromorphically multivalent functions. We presented some results concerning the geometric properties of the given operator connecting with the Janowski function in the open unit disk. Our results showed under some conditions, the given operator converges to the Janowski function. 
Availability of data and material: Not applicable.

Conflicts of Interest: The authors declare no conflict of interest.

Funding: Not applicable.

Author Contributions: All authors contributed equally and significantly in writing this article. All authors read and approved the final manuscript.

Acknowledgments: The authors would like to thank the reviewers for the deep comments to improve our work. Also, we express our thanks to editorial office for their advice.

\section{References}

[1] G.W. Leibniz, Letter from Hanover, Germany to G.F.A. L'Hospital, September 30, 1965, Leibniz Mathematische Schriften, Olms-Verlag, Hildesheim, Germany, 1849 (1962).

[2] E. Capelas de Oliveira, J. A. Tenreiro Machado, A Review of Definitions for Fractional Derivatives and Integral, Math. Probl. Eng., 238459 (2014).

[3] R. Khalil, M. Al Horani, A. Yousef, M. Sababheh, A new definition of fractional derivative, J. Comput. Appl. Math. 264 (2014).

[4] U. Katugampola, A new fractional derivative with classical properties, (2014), arXiv:1410.6535v2

[5] T. Abdeljawad, On conformable fractional calculus, J. Comput. Appl. Math. 279 (2015).

[6] D.R. Anderson, D.J. Ulness, Newly defined conformable derivatives, Adv. Dyn. Syst. Appl 10.2 (2015).

[7] Y. Li, K. H. Ang , G. Chong, PID control system analysis and design, IEEE Control Syst. Mag., 26,1 (2006).

[8] R. W. Ibrahim, J. M. Jahangiri, Conformable differential operator generalizes the Briot-Bouquet differential equation in a complex domain, AIMS Math 4,6 (2019).

[9] R.W. Ibrahim, D. Baleanu, On a combination of fractional differential and integral operators associated with a class of normalized functions, AIMS Mathematics, 6,4 (2021).

[10] R.W. Ibrahim, D. Baleanu, On quantum hybrid fractional conformable differential and integral operators in a complex domain, Revista de la Real Academia de Ciencias Exactas, Fìsicas y Naturales. Serie A. Matemáticas 115, 1 (2021).

[11] R. W. Ibrahim, Geometric properties of the differential shift plus complex Volterra operator, Asian-European Journal of Mathematics 11,1 (2018).

[12] R. W. Ibrahim, On a class of analytic functions generated by fractional integral operator." Concrete Operators, 4, 1 (2017).

[13] Y. Komatu, Note on the theory of conformal representation by meromorphic functions II, Proc. Japan Acad. 21 (1945).

[14] W. K. Hayman, Meromorphic Functions", Oxford Math. Monographs, Oxford, 1964.

[15] W. Rogosinski, On subordination functions, Proc. Cambridge Phil. Soc. 35,1 (1939).

[16] W. Janowski, Some extremal problems for certain families of analytic functions I, Ann. Polon. Math. 28 (1973).

[17] J. M. Jahangiri, H. Silverman, E. M. Silvia, Inclusion relations between classes of functions defined by subordination, J. Math. Anal. Appl. 151 (1990).

[18] S. S. Miller, P. T. Mocanu. Differential subordinations: theory and applications. CRC Press, 2000.

[19] P. L. Duren, Univalent functions, Grundlehren der Mathematischen Wissenschaften, 259, (1983), Springer-Verlag, ISBN 0387-90795-5. 